In a similar manner we may secure three relations between the constants by choosing a straight line $C_{1}$ to be the degenerate first Laplacian transform and a point $v=v_{0}$ on the curve $C^{\prime}$. The six relations are sufficient to determine the six arbitrary constants. We may therefore state the following conclusion.

Choose two non-rectilinear but otherwise arbitrary analytic curves $C$ and $C^{\prime}$ intersecting in a point $P$ and having distinct tangents $T$ and $T^{\prime}$ at $P$. Choose an arbitrary straight line $C_{-1}$ intersecting $T$ and another line $C_{1}$ intersecting $T^{\prime}$. There exists one and only one net which contains the curves $C$ and $C^{\prime}$ and which moreover has $C_{1}$ and $C_{-1}$ for its degenerate first and minus first Laplacian transforms.

The family of curves $v=$ const. may be obtained from the curve $C$ by projective transformations. Similarly, the family $u=$ const. may be obtained from $C^{\prime}$.

The University OF OKLAHoma

\title{
SOME PROPERTIES OF UPPER SEMI-CONTINUOUS COLLECTIONS OF BOUNDED CONTINUA*
}

BY W. A. WILSON

1. Iniroduction. If $T=\{t\}$ denotes a closed set of points and with each point $t$ there is associated a unique bounded continuum $X$ (or $X_{t}$ ) in such a way that (a) $X_{t} \cdot X_{t^{\prime}}=0$ if $t \neq t^{\prime}$, (b) at each point $t=\tau$ of $T$ the upper closed limit of $X_{t}$ as $t \rightarrow \tau$ is a part of $X_{\tau}$, we say that $X=f(t)$ is an upper semicontinuous function in $T$. The collection of continua $\{X\}$ is also known as an upper semi-continuous collection of continua. These aggregates have been discussed by various writers here and abroad and enjoy numerous interesting properties.

R. L. Moore, in particular, has given an extensive treat-

* Presented to the Society, February 25, 1928. 
ment of the subject $\dagger$ and among other things has shown that, if the continua $\{X\}$ all lie in a plane but none of them cuts the plane, then the complement of $M=\Sigma[X]$ is a simply connected region whose frontier is a part of $M$ if $T$ is a simple arc and the complement of $M$ is two simply connected regions whose frontiers are parts of $M$ if $T$ is a circumference. It is easily seen by examples that the frontiers of the complementary regions need not coincide with $M$ in either case; it is the purpose of this note to give the conditions under which they do. $\ddagger$

To this end we define $X=f(t)$ as a minimal upper semicontinuous function in $T$ if there exists no upper semicontinuous function $Y=g(t)$ such that at every point $t$, $Y \subset X$, and at some point $Y \neq X$. If $T$ denotes the interval $(-1 \leqq t \leqq 1)$ and in some plane we let $X_{t}$ be the point $(t, \sin (1 / t))$ when $t \neq 0$ and the point set $x=0,-1 \leqq y \leqq 2$ when $t=0$, then $X=f(t)$ is an upper semi-continuous function which is not a minimal function, but becomes so if we replace $X_{0}=f(0)$ by the set $x=0,-1 \leqq y \leqq 1$. An example of a minimal upper semi-continuous function where no $X$ is a point is given by the author in this Bulletin, vol. 32, p. 679 .

2. Notation. The following notation will be convenient. If $X=f(t)$ in $T$ and $M=\Sigma[X]$ we write $M=F(T)$. If $T$ is a bounded continuum and $f(t)$ is upper semi-continuous, it is obvious that $M$ is a bounded continuum; in this case we say that $X$ is an element of $M$.

If $T$ is a simple arc $a b, M=F(a b)$ will be called a generalized arc, or simply an arc if no confusion is caused. This may be denoted by $X_{a} X_{b}$ and the elements $X_{a}$ and $X_{b}$ will be called the ends. Likewise, $M-\left(X_{a}+X_{b}\right)$ is

$\dagger$ R. L. Moore, Concerning upper semi-continuous collections of continua, Transactions of this Society, vol. 27, pp. 416-428.

$\ddagger$ The attention of the reader is directed to an article by $C$. Kuratowski, Sur la structure des frontières communes a deux régions, Fundamenta Mathematicae, vol. 12 (1928), pp. 20-42, of which an advance copy was received while this paper was in press. Although Kuratowski's article is concerned chiefly with the converse problem, the reader will note a certain degree of similarity between the two papers. 
called a (generalized) open arc and denoted by $X_{a}{ }^{*} X_{b}{ }^{*}$.

If $T$ is a circumference $C, M=F(C)$ is called a (generalized) simple closed curve. Obviously any two elements $X_{1}$ and $X_{2}$ divide $M$ into two arcs having $X_{1}$ and $X_{2}$ as end elements and no other common points.

The plane will be denoted throughout by $Z$.

3. Certain Corollaries. Certain properties of the sets under consideration are either corollaries of Moore's work or are so easily demonstrated that their proofs are omitted.

(a) If $M=F(a b)$ is a generalized arc in a plane $Z$, no subcontinuum of $M$ separates $X_{a}$ from $X_{b}$ unless some element of $M$ does.

(b) If $M=F(C)$ is a generalized simple closed curve in a plane, no two elements of $M$ are separated by a sub-continuum of $M$.

These are readily proved with the aid of a theorem of Janiszewski. $\dagger$

(c) In a plane $Z$ let $M=F(a b)$ be a generalized arc and no element of $M$ separate $X_{a}$ from $X_{b}$, or let $M=F(C)$ be a generalized simple closed curve. Then there is not more than one element $X$ such that a bounded component of $Z-X$ contains $M-X$.

(d) In a plane $Z$ let $M=F(a b)$ be a generalized arc and no element of $M$ separate $X_{a}$ from $X_{b}$, or let $M=F(C)$ be a generalized simple closed curve. For each element $X$ let the component of $Z-X$ containing $M-X$ be unbounded. Let $Y$ be the union of $X$ and the components of $Z-X$ containing no points of $M$. Then $Y=g(t)$ is upper semi-continuous in $a b$ or $C$, respectively.

4. Lemma. Let $M=F(C)$ be a generalized simple closed curve in a plane $Z$. Then $Z-M$ has two components whose frontiers have points in every element of $M$ and every other component has a frontier which is a part of some element.

$\dagger$ Z. Janiszewski, Sur les coupures du plan faites par des continus, Prace Matematyczno-Fizyczne, vol. 26, Theorem A. See also R. L. Moore, Concerning the prime parts of certain continua which separate the plane, Proceedings of the National Academy of Sciences, vol. 10, p. 173. 
Proof. This is really a corollary of R. L. Moore's work. By $\S 3$ (c) there is at most one element $X$ such that a bounded component of $Z-X$ contains $M-X$. Since inversion with respect to a point within this component will make its image unbounded, there is no loss in generality if we assume that for every element $X$ the unbounded component of $Z-X$ contains $M-X$.

Defining $Y=g(t)$ as in $\S 3(\mathrm{~d})$, it follows from this reference that $N=\Sigma[Y]$ is a generalized simple closed curve no element of which separates $Z$. In this case Moore has shown (loc. cit., Theorem 11) that $Z-N$ consists of two components $R$ and $S$, and the frontier of each of these has at least one point on every element $Y$. It is readily seen that these are also components of $Z-M$. Hence the first part of the lemma is proved.

That the frontier of each of the other components of $Z-M$ is a part of some element $X$ is a consequence of the definition of $Y=g(t)$.

Definition. The components of $Z-M$ which have frontier points on every element of $M$ will be called principal components.

Corollary. Let $C=\{t\}$ be a circumference, let $X=f(t)$ be an upper semi-continuous function defined over $C$, and let $M=\Sigma[X]$ lie in the plane $Z$. If $M$ is the common frontier of two components of $Z-M$, then $f(t)$ is a minimal upper semicontinuous function.

Proof. Let $R$ and $S$ be the components of $Z-M$ having the frontier $M$. If the theorem is not true, let $Y=g(t)$ be upper semi-continuous over $C$, let $Y \subset X$ for every $t$, and let $Y \neq X$ for some $t$. Let $N=\Sigma[Y]$.

By the above lemma, $Z-N$ has two principal components $R^{\prime}$ and $S^{\prime}$. Since $M$ is an irreducible cut of $Z$ between points of $R$ and $S$ and $N$ is a proper part of $M, R$ and $S$ lie in the same component of $Z-N$, and this must contain all the points of $M-N$. Suppose that $S^{\prime} \cdot(R+S)=0$. Then the frontier of $S^{\prime}$ is a part of some element of $M$, as $S^{\prime}$ is a com- 
ponent of $Z-M$. This is a contradiction, since $N$ contains points of every element of $M$.

5. Lemma. Let $M=F(C)$ be a generalized simple closed curve in a plane $Z$, and let $R$ be one of the principal components of $Z-M$. Let $a$ and $b$ be points of $M$ accessible from $R$ and lying on different elements $A$ and $B$ of $M$. Let $A$ and $B$ divide $M$ into the arcs $M_{1}$ and $M_{2}$. If $F$ is the frontier of $R, F=H+K$, where $H$ and $K$ are sub-continua of $M_{1}$ and $M_{2}$, respectively, joining $a$ and $b$.

Proof. Let $m$ be a point of $R$ and $m a$ and $m b$ be simple arcs lying in $R$ except for the points $a$ and $b$ and having only $m$ in common. R. L. Moore has shown (loc. cit., p. 423) that the arc $a b=m a+m b$ divides $R$ into two simply connected regions $R_{1}$ and $R_{2}$ such that their frontiers are parts of $M_{1}+a^{*} b^{*}$ and $M_{2}+a^{*} b^{*}$, respectively.

Let $H$ and $K$, respectively, denote those points of these frontiers not on $a^{*} b^{*}$; then $H \subset M_{1}, K \subset M_{2}$. Since the frontier of $R_{1}$ is a continuum, every point of $H$ can be joined to $a$ or $b$ by a sub-continuum of $H$. If $H$ is not a continuum, $H=H_{1}+H_{2}$, where $H_{1}$ and $H_{2}$ are continua containing $a$ and $b$, respectively, and $H_{1} \cdot H_{2}=0$. This hypothesis would give a contradiction by the theorem of Janiszewski referred to earlier, for neither $a b, H_{1}$, nor $H_{2}$ separates $R_{1}$ from $R_{2}$, while $(a b) \cdot H_{1}=a,(a b) \cdot H_{2}=b$, and $\left(a b+H_{1}\right) \cdot\left(a b+H_{2}\right)=a b$. Thus $H$, and in like manner $K$, is a continuum.

Now $F \supset H+K$. On the other hand every frontier point of $R$ is necessarily one of $R_{1}$, or of $R_{2}$, or of both. Hence $F=H+K$.

6. Theorem. Let $C=\{t\}$ be a circumference, let $X=f(t)$ be a minimal upper semi-continuous function defined over $C$, and let $M=\Sigma[X]$ lie in the plane $Z$. Then $M$ is the frontier of two components of $Z-M$ and the frontier of each of the remaining components is a part of some element of $M$.

Proof. The last assertion is a restatement of a previous result. (See §4.) Let $R$ and $S$ be the principal components of $Z-M$, and let $F$ be the frontier of one of them, say $R$. Since 
accessible points are everywhere dense in $F$ and $F$ contains at least one point in every element $X$, there is an everywhere dense set of points $t$ each of whose corresponding elements contains an accessible point of $F$.

Orient the points of $C$, let $\tau$ be a fixed point $t$, and let $\left\{t_{i}\right\}$ and $\left\{t_{i}{ }^{\prime}\right\}$ be sequences of points $\{t\}$ such that $t_{1}<t_{2}<\cdots \rightarrow \tau, t_{1}{ }^{\prime}>t_{2}{ }^{\prime}>\cdots \rightarrow \tau$, and for each $t_{i}$ and $t_{i}{ }^{\prime}$ the corresponding $X_{i}$ or $X_{i}{ }^{\prime}$ contains an accessible point of $F$.

Let $M_{i}$ be the arc of $M$ joining $X_{i}$ and $X_{i}{ }^{\prime}$ and containing $X_{\tau}=f(\tau)$. Obviously $X_{\tau}=\Pi_{1}^{\infty}\left[M_{i}\right]$. By the previous lemma $M_{i}$ contains a sub-continuum $F_{i}$ of $F$ joining $X_{i}$ and $X_{i}{ }^{\prime}$, and $\left(F-F_{i}\right) \cdot X_{\tau}=0$. Moreover, $\Pi_{1}^{\infty}\left[F_{i}\right]$ is a continuum.

But $F_{i} \subset M_{i}$; hence $\Pi\left[F_{i}\right] \subset X_{\tau}$. As $\left(F-F_{i}\right) X_{\tau}=0, F \cdot X_{\tau}$ c $F_{i}$, whence $F \cdot X_{r}=\Pi\left[F_{i}\right]$. Thus we have shown that for each $t, Y_{t}=F \cdot X_{t}$ is a continuum.

On the other hand, $\varlimsup_{t \rightarrow \tau}, Y_{t} \subset F \cdot X_{\tau} \subset Y_{\tau}$. For $Y_{t} \subset F$ $Y_{t} \subset X_{t}$, and $X_{t}=f(t)$ is upper semi-continuous. Thus $Y_{t}=g(t)$ is upper semi-continuous. But $f(t)$ is a minimal upper semi-continuous function. Hence for every $t, Y_{t}=X_{t}$ and so $F=M$.

Corollary. Let $M$ satisfy the hypotheses of the above theorem, let $A$ and $B$ be any two elements of $M$, and $M_{1}$ and $M_{2}$ the complementary arcs of $M$ thus determined. Then $M=H_{1}$ $+H_{2}$, where $H_{1} \subset M_{1}, H_{2} \subset M_{2}, H_{1} \cdot H_{2}=\alpha+\beta$, where $\alpha \subset A$, $\beta \subset B$, and both $H_{1}$ and $H_{2}$ are continua irreducible between $\alpha$ and $\beta$.

Proof. Let $M=H_{1}+H_{2}$ be an irreducible decomposition* of $M$ such that $H_{1} \subset M_{1}$ and $H_{2} \subset M_{2}$. Then $H_{1} \supset M_{1}-(A+B)$ and $H_{2} \supset M_{2}-(A+B)$. By the above theorem $M$ is an irreducible cut of the plane between a point of $R$ and one of $S$. By a theorem proved elsewhere $H_{1}$ and $H_{2}$ are both irreducible between $\alpha=A \cdot H_{1} \cdot H_{2}$ and $\beta=B \cdot H_{1} \cdot H_{2}$.

* The decomposition $M=H_{1}+H_{2}$ is called irreducible if $H_{1}$ and $H_{2}^{2}$ are continua and there exists no proper sub-continuum $K$ of $H_{1}$ or $H_{2}$ such that $M=K+H_{2}$ or $M=H_{1}+K$, respectively.

$\dagger$ W. A. Wilson, On irreducible cuts of the plane between two points, Annals of Mathematics, vol. 29, $\$ 9$. 
7. Lemma. Let $M=F(a b)$ be a generalized arc lying in a plane $Z$ and let no element of $M$ separate $X_{a}$ from $X_{b}$. Then $Z-M$ has one component whose frontier has points on every element of $M$ and every other component has a frontier which is a part of some elements.

Proof. This is a corollary of a theorem by R. L. Moore (loc. cit., Theorem 9). It is proved in the same manner as the lemma of $\S 4$.

8. Theorem. Let the aggregate $\{t\}$ be a simple arc ab, let $X=f(t)$ be a minimal upper semi-continuous function defined over $a b$, and let $M=\Sigma[X]$ lie in the plane $Z$, while no element of $M$ separates $X_{a}$ from $X_{b}$. Then $M$ is a continuum irreducible between $X_{a}$ and $X_{b}$.

Proof. By $\$ 7$ there is one component $R$ of $Z-M$ which has frontier points on every element of $M$. Since accessible points are everywhere dense, there is a decreasing sequence $\left\{t_{i}\right\}$ where $t_{i} \rightarrow a$ and an increasing sequence $\left\{t_{i}{ }^{\prime}\right\}$ where $t_{i}{ }^{\prime} \rightarrow b$, such that $X_{i}=f\left(t_{i}\right)$ and $X_{i}{ }^{\prime}=f\left(t_{i}{ }^{\prime}\right)$ contain accessible points $x_{i}$ and $x_{i}{ }^{\prime}$, respectively.

Let $x_{i} x_{i}{ }^{\prime}$ be a simple arc lying in $R$ except for the end points. Let $u$ run over a circumference $C$. Let the segment $t_{i} t_{i}{ }^{\prime}$ of $a b$ be homeomorphic with an arc $c d$ of $C$ in such a way that $t_{i}$ corresponds to $c$ and $t_{i}{ }^{\prime}$ to $d$. Let the arc $x_{i} x_{i}{ }^{\prime}$ be homeomorphic to the complementary arc $d c=\overline{C-c d}$ in such a way that $x_{i}$ corresponds to $c$ and $x_{i}{ }^{\prime}$ to $d$. Now define the function $Y=g(u)$ as follows. If $u=c, Y$ is a subcontinuum of $X_{i}$ irreducible about $x_{i}$ and $\varlimsup_{\lim } X_{t}$ as $t \rightarrow t_{i}$ in $t_{2} t_{i}{ }^{\prime}$; if $u=d, Y$ is a sub-continuum of $X_{i}{ }^{\prime}$ irreducible about $x_{i}{ }^{\prime}$ and $\overline{\lim } X_{t}$ as $t \rightarrow t_{i}{ }^{\prime}$ in $t_{i} t_{i}{ }^{\prime}$; if $u$ is any other point of $c d$ and $t$ is the corresponding point of $t_{i} t_{i}{ }^{\prime}, Y=X_{t}=f(t)$; if $u$ is a point of $d c-(c+d), Y$ is the corresponding point $x$ of the simple arc $x_{i} x_{i}{ }^{\prime}$. It is readily seen that $Y=g(u)$ is a minimal upper semi-continuous function and that $N=\Sigma[Y]=G(C)$ is a generalized simple closed curve.

Now $Y_{c}$ and $Y_{d}$ determine two generalized arcs $N_{1}=G(d c)$ and $N_{2}=G(c d)$ having these elements as ends and no other 
common points. If we set $H_{1}$ equal to the simple arc $x_{i} x_{i}{ }^{\prime}$ and $H_{2}=N-x_{i} x_{i}{ }^{\prime}+x_{i}+x_{i}{ }^{\prime}$, it is evident that $H_{1} \subset N_{1}$, $H_{2} \subset N_{2}$, and that $N=H_{1}+H_{2}$ is an irreducible partition of $N$. Then by $\S 6$, Corollary, $H_{2}$ is irreducible between $x_{i}$ and $x_{i}{ }^{\prime}$. As $H_{2} \supset N_{2}-\left(Y_{c}+Y_{d}\right), H_{2} \supset X_{i} X_{i}{ }^{\prime}-\left(X_{i}+X_{i}{ }^{\prime}\right)$. That is, any sub-continuum of the $\operatorname{arc} X_{i} X_{i}{ }^{\prime}$ joining the end elements contains all elements between them. Hence any sub-continuum of $M$ joining $X_{a}$ and $X_{b}$ contains every point of all the elements between $X_{i}$ and $X_{i}{ }^{\prime}$. As $t_{i} \rightarrow a$ and $t_{i}{ }^{\prime} \rightarrow b$, this means that it contains every point of $M-\left(X_{a}+X_{b}\right)$. But, since $X=f(t)$ is a minimal upper semicontinuous function, $M=\overline{M-\left(X_{a}+X_{b}\right)}$. Hence $M$ is irreducible between $X_{a}$ and $X_{b}$.

Corollary. Let the aggregate $\{t\}$ be a simple arc ab, let $X=f(t)$ be a minimal upper semi-continuous function defined over $a b$, and let $M=\Sigma[X]$ lie in the plane $Z$, while no element of $M$ separates $X_{a}$ from $X_{b}$. Then $M$ is the frontier of one component of $Z-M$ and the frontier of each of the remaining components is a part of some element of $M$.

Proof. By $\S 7$ there is one component of $Z-M$ whose frontier has points on every element of $M$. Since this frontier is a continuum joining $X_{a}$ and $X_{b}$ and is a part of $M$, it must coincide with $M$ by the above theorem. The last part of the corollary is merely restated from $\$ 7$.

YALE UNIVERSITY 